\title{
INTEGRATION OF MULTITEMPORAL SENTINEL-1 AND SENTINEL-2 IMAGERY FOR LAND-COVER CLASSIFICATION USING MACHINE LEARNING METHODS
}

\author{
D. Dobrinić ${ }^{1 *}$, D. Medak ${ }^{1}$, M. Gašparović ${ }^{2}$ \\ ${ }^{1}$ Faculty of Geodesy, Chair of Geoinformatics, University of Zagreb, 10000 Zagreb, Croatia - (ddobrinic, dmedak)@geof.unizg.hr \\ ${ }^{2}$ Faculty of Geodesy, Chair of Photogrammetry and Remote Sensing, University of Zagreb, 10000 Zagreb, Croatia - \\ mgasparovic@geof.unizg.hr
}

Commission I, WG I/3

KEY WORDS: Land-Cover Classification, Multitemporal Analysis, Random Forest, SAR, Sentinel-1, Sentinel-2, XGBoost.

\begin{abstract}
:
Using space-borne remote sensing data is widely used for land-cover classification (LCC) due to its ability to provide a big amount of data with a regular temporal revisit time. In recent years, optical and synthetic aperture radar (SAR) imagery have become available for free, and their integration in time series have improved LCC. This research evaluates the classification accuracy using multitemporal (MT) Sentinel-1 (S1) and Sentinel-2 (S2) imagery. Pixel-based LCC is made for S1 and S2 imagery, and for a combination of both datasets with Random Forest (RF) and Extreme Gradient Boosting (XGBoost; XGB). The extent of the study area, is located in the south-east of France, in Lyon. Regardless of LCC using single-date or MT data, the highest classification results were achieved with integrated S1 and S2 imagery and XGB method, whereas overall accuracy (OA) and Kappa coefficient (Kappa) increased from $85.51 \%$ to $91.09 \%$, and from 0.81 to 0.88 , respectively. Furthermore, the integration of MT imagery significantly improved the classification of urban areas and reduced misclassification between forest and low vegetation. In this paper, in terms of the pixel-based classification, XGB produced slightly better results than RF, and outperformed it in terms of computational time. This research improved LCC with integration of radar and optical MT imagery, which can be useful for areas hampered by a frequent cloud cover. Future work should use the aforementioned data for specific applications in remote sensing, as well as evaluate the classification performance with different approaches, such as neural networks or deep learning.
\end{abstract}

\section{INTRODUCTION}

Land-cover classification (LCC) is significant for monitoring urban growth, agricultural planning, and deforestation (Souza, Jr et al., 2013; Veloso et al., 2017; Zakeri et al., 2017). Satellite imagery acquired from remote sensing (RS) is widely used in LCC and monitoring owing to a big amount of spatial data with a daily revisit time. The usual way of performing classification tasks is the use of optical satellite imagery. Optical RS uses the sun as an external source of irradiance; however, the acquisition of optical imagery may be limited if the cloud layer is large (Sun et al., 2019). Being an active microwave sensor, synthetic aperture radar (SAR) can provide data acquisition that is independent of solar illumination and cloud cover, as microwave radiation penetrates through clouds. SAR data is sensitive to the surface roughness, textural and dielectric properties of land objects (Feng et al., 2019).

For LCC, many studies preferred optical data to SAR imagery, because of a better understanding of the links between the observations (Immitzer et al., 2012; Gašparović et al., 2018; Noi and Kappas, 2018). In recent years, optical and SAR imagery have become available for free, and their integration in time series have improved LCC. Van Tricht et al. (2018) investigated the possibility of crop mapping using joint radar (Sentinel-1; S1) and optical (Sentinel-2; S2) data. The integration of S1 and S2 imagery, led to higher classification accuracies compared to optical-only classification. Sonobe et al. (2017) evaluated the suitability of S1 and S2 data for the classification of various crop types. Classification for a set of six crop types on five S1 and one S2 imagery with a Random Forest (RF) algorithm achieved overall accuracy (OA) of $95.7 \%$. The research showed a remarkable potential for crop classification. Gómez (2017) combined S1 and S2 data for LCC. For a pixel-based classification of six land-cover classes with RF algorithm achieved OA was $84.33 \%$, and Kappa of 0.81 . For the pixelbased approach, the used data was S1, S2, and vegetation indices.

The aforementioned research used data from Sentinel satellites developed within the Copernicus Programme. European Space Agency (ESA) provided free and open access to S1 radar and S2 optical data, with almost global data availability with the revisit frequency of 6 and 5 days, respectively (Van Tricht et al., 2018).

Besides highly spatial, temporal and spectral resolutions of the data, machine learning methods are fundamental for developing LCC maps over a large area within short acquisition window. Classifiers such as artificial neural networks (ANN), Support Vector Machine (SVM), or RF outperform traditional parametric approaches with their ability to deal with noise and unbalanced datasets (Abdullah et al., 2019). In this research, used algorithms were RF, and extreme gradient boosting (XGBoost; XGB). RF is a robust classifier that avoids overfitting through bootstrapping and provides good classification results and computer processing time (Waske and Braun, 2009; Niculescu et al., 2018). XGB is an implementation of gradient boosted decision trees developed by Chen and Guestrin (2016). In recent research for LCC applications, XGB slightly outperformed RF and SVM with increased processing time (Man et al., 2018; Hirayama et al., 2019). When the

* Corresponding author 
number of samples is large, SVM needs a lot of machine memory leading to increased computation time, so this algorithm was not used in this research (Mountrakis et al., 2011).

The purpose of this paper is (1) to evaluate how classification accuracy depends on the multitemporal input source (optical data, radar data, or a combination of both) at a pixel-level and (2) to evaluate the performance of the machine learning methods for producing LCC maps.

\section{STUDY AREA AND DATA}

\subsection{Study area}

For the research, the city of Lyon, which is located in the southeast of France, was chosen (Figure 1). The city is surrounded by the rivers Rhone and Saone, and it is the third most populated city in France. The characteristics of a study area is a mild climate with an average temperature of $11.6^{\circ} \mathrm{C}$. The average annual precipitation is $763 \mathrm{~mm}$. For this research, almost 1200 $\mathrm{km}^{2}$ area $(30 \mathrm{~km} \mathrm{x} 40 \mathrm{~km})$ was examined, which includes landcover classes such as water, bare soil, forest, built-up and low vegetation.
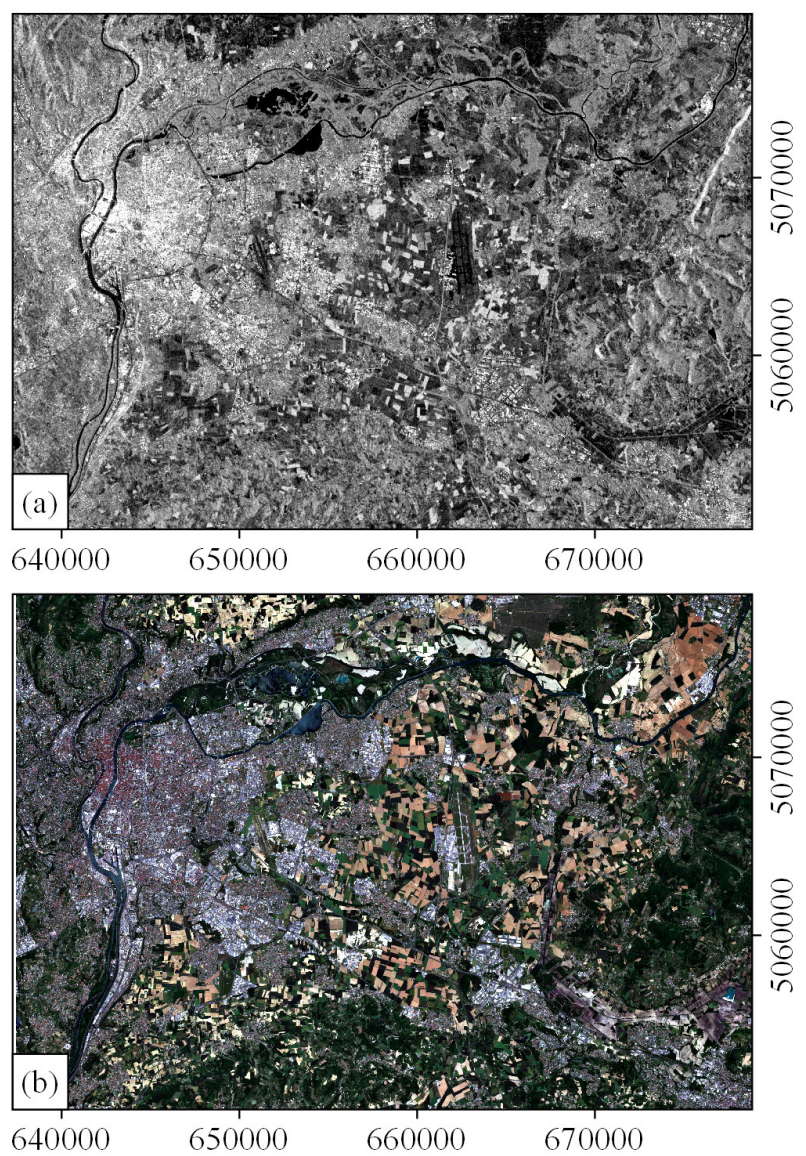

Projection: WGS 1984/UTM Zone 31 N; EPSG: 32631

Figure 1. Overview of the study area on (a) S1 imagery (VH dB values; 04-06-2019) and (b) S2 imagery ("true colour" composite (B04-B03-B02); sensing date: 02-06-2019).

\subsection{Data}

Because of the availability of S1 and S2 data, both multitemporal and multisensor Sentinel data were used for LCC. S1 is an imaging radar satellite whose constellation comprises two satellites: S1A and S1B. Both satellites carry a C-band $(\sim 5.55 \mathrm{~cm})$, capable of providing dual polarisation observations in several measuring modes (Torres et al., 2012). For this research, three imagery of S1 GRDH (ground range detected in high resolution) products were used with a spatial resolution of $10 \mathrm{~m}$. S1 Level-1 imagery were selected according to the date proximity in relation to the cloud-free S2 imagery (Table 1).

\begin{tabular}{|l|c|c|c|}
\hline & \multicolumn{3}{|c|}{ Sentinel-1 } \\
\hline $\begin{array}{l}\text { Acquisition } \\
\text { date }\end{array}$ & $23-05-2019$ & $04-06-2019$ & $10-06-2019$ \\
\hline $\begin{array}{l}\text { Acquisition } \\
\text { orbit }\end{array}$ & ASC & ASC & ASC \\
\hline $\begin{array}{l}\text { Orbit } \\
\text { number }\end{array}$ & 27358 & 27533 & 16637 \\
\hline Polarisation & \multicolumn{3}{|c|}{ VV, VH } \\
\hline
\end{tabular}

Table 1. Characteristics of the S1 data; vertical-vertical (VV) and vertical-horizontal $(\mathrm{VH})$ polarizations.

S2 also consists of two identical polar-orbiting satellites, and it provides high resolution multispectral optical imagery within 13 spectral bands. For this research, three optical S2 (Level-2A) scenes were selected for LCC. We selected temporal imagery with zero cloud coverage (Table 2). Spectral bands in the visible and near-infrared spectrum, i.e., Blue (B02), Green (B03), Red (B04), and the Near-Infrared band (B08) with an identical spatial resolution of $10 \mathrm{~m}$ as $\mathrm{S} 1$ were used.

\begin{tabular}{|l|c|c|c|}
\hline & \multicolumn{3}{|c|}{ Sentinel-2 } \\
\hline $\begin{array}{l}\text { Acquisition } \\
\text { date }\end{array}$ & $23-05-2019$ & $02-06-2019$ & $17-06-2019$ \\
\hline $\begin{array}{l}\text { Sun zenith } \\
\text { angle }\left[{ }^{\circ}\right]\end{array}$ & 26.88 & 25.46 & 24.59 \\
\hline $\begin{array}{l}\text { Orbit } \\
\text { number }\end{array}$ & 20454 & 20597 & 11903 \\
\hline $\begin{array}{l}\text { Spectral } \\
\text { bands }\end{array}$ & \multicolumn{3}{|c|}{ B02, B03, B04, B08 } \\
\hline
\end{tabular}

Table 2. Characteristics of the S2 imagery used in this research.

\section{METHODS}

\subsection{Sentinel-1 data pre-processing}

After downloading the S1 imagery from the ESA's Sentinel Scientific Hub, SAR data pre-processing was implemented using S1 Toolbox provided by the ESA. S1 Level-1 products are not radiometrically corrected by default, and therefore digital pixel values need to be converted to radiometrically calibrated SAR backscatter (Filipponi, 2019). The radiometric calibration is performed by calculating the sigma naught $\left(\sigma^{0}\right)$. Speckle noise is inevitable in the SAR imagery, owing to the coherent mode of backscattered signal processing (Oliver, 1991). Therefore, speckle filtering is necessary for most SAR image analysis. Along with many developed spatial speckle filters for speckle suppression (Shi and Fung, 1994), a 5 x 5 Frost filter was applied to each image. The Frost filter reduces 
speckle noise by using local statistics in order to efficiently preserve edges in radar imagery (Frost et al., 1982). The aforementioned filter was already used in similar research for LCC using multitemporal (MT) SAR imagery (Waske and Braun, 2009; Maghsoudi et al., 2012), and hence, Frost filter was applied on single-date S1 imagery which was then stacked together. Level-1 GRD imagery are not considered for the geometric distortions caused by terrain topography. Therefore, Range Doppler terrain correction operator is applied on the S1 imagery in order to improve or evaluate the geopositioning accuracy (Dobrinić et al., 2018; Gašparović et al., 2019), and projecting the scenes in WGS 1984/UTM Zone $31 \mathrm{~N}$. Before the pixel-based LCC, the $\sigma^{0}$ values were transformed to $\mathrm{dB}$ values through its logarithmic form, as shown in Equation (1) (López-Caloca et al., 2018):

$$
\sigma_{d b}^{0}=10 \log _{10} \sigma^{0}
$$

\subsection{Land-cover classification algorithms}

For the supervised pixel-based classification, RF and XGB classifiers were tested using $\mathrm{R}$ version 3.6.0 ( $\mathrm{R}$ Core Team, 2016).

$\mathrm{RF}$ is a tree-based algorithm that is created from a large number of individual decision trees (Breiman, 2001). Classifier randomly selects subset features using bagging method, and it is relatively robust to outliers and noise (Rodriguez-Galiano et al., 2012). RF has two hyperparameters: number of trees to grow within the model (ntree) and how many variables are available for selection at a node split (mtry). A research by Kulkarni et al. (2012) evaluated that mtry hyperparameter has a larger impact on classification accuracy than ntree parameter.

$\mathrm{XGB}$, as an ensemble tree-boosting model, converts weak learners into strong learners. Weak learners are added until no further improvements can be made, and by using a gradient descent algorithm, the loss of the model is minimized (Chen and Guestrin, 2016). XGB has many hyperparameters that need to be optimized, as described by Man et al. (2018).

\subsection{Accuracy assessment}

In this research, the land-cover classes were selected using common categories (Table 3) described in similar studies (Clerici et al., 2017; Gašparović et al., 2018). The classification accuracy was assessed based on the error matrix (Foody, 2010). Overall, 800 polygons were divided into training $(70 \%)$ and validation $(30 \%)$ subsets. From the error matrix, the accuracy for the LCC was assessed using the overall accuracy (OA) and Kappa coefficient (Kappa). For land-cover classes, the User's accuracy (UA) and Producer's accuracy (PA) were computed.

\begin{tabular}{|c|c|c|}
\hline ID & Class & Number of samples \\
\hline 1 & Water & 140 \\
\hline 2 & Bare soil & 160 \\
\hline 3 & Forest & 160 \\
\hline 4 & Built-up & 200 \\
\hline 5 & Low vegetation & 160 \\
\hline
\end{tabular}

Table 3. Overview of the land-cover classes used in this research.

\section{RESULTS AND DISCUSSION}

The results of the LCC using the two machine learning methods described in Section 3 are shown here. In Section 4.1, results using the single-date S1 and S2 imagery are discussed, and in Section 4.2, results using the MT S1, and S2 imagery are discussed.

\subsection{Land-cover classification on a single-date S1 and S2 imagery}

In order to evaluate how classification accuracy changes on MT imagery, firstly, classification was done using single-date S1 and S2 imagery. For S1 and S2 imagery, June $4^{\text {th, }}$ and June $2^{\text {nd }}$, 2019, were chosen as reference dates, respectively. OA and Kappa values for S1, S2, and using the combination of S1 and S2 are shown in Table 4.

\begin{tabular}{|l|c|r|r|r|r|r|}
\hline Sensor & \multicolumn{2}{|c|}{ S1 } & \multicolumn{2}{c|}{ S2 } & \multicolumn{2}{c|}{ S1+S2 } \\
\hline Method & RF & XGB & RF & XGB & \multicolumn{1}{c|}{ RF } & \multicolumn{1}{c|}{ XGB } \\
\hline $\begin{array}{l}\text { OA } \\
{[\%]}\end{array}$ & 70.41 & 72.02 & 84.17 & 83.75 & 85.47 & 85.51 \\
\hline Kappa & 0.61 & 0.63 & 0.80 & 0.79 & 0.81 & 0.81 \\
\hline
\end{tabular}

Table 4. OA and Kappa values of RF and XGB, as applied to the single-date S1 and S2 imagery.

For the S1 classification, XGB performed better than RF with OA values of $72.02 \%$, and $70.41 \%$, respectively, whereas in the S2 classification, RF achieved higher accuracy metrics than the XGB method, with an OA of $84.17 \%$, and Kappa of 0.80 . In the single-date SAR land-cover classification, speckle noise presents a challenging task in order to reduce speckle for quality image interpretation and further analysis (Xiao et al., 2003). Optical imagery, like S2, has already proven for the LCC applications, so we wanted to investigate the integration of radar and SAR data, similar to the research of Van Tricht et al., (2018). XGB performed slightly better than RF for the combined S1 and S2 classification with an OA of $85.51 \%$ and Kappa 0.81. Similar to the LCC results obtained in Hirayama et al. (2019), XGB slightly outperformed RF. Irrespective of the classifier used for combined $\mathrm{S} 1$ and $\mathrm{S} 2$ pixel-based classification, increased OA, and Kappa values overlap with similar research in LCC (Gómez, 2017; Abdi, 2019). For better discrimination of the land-cover classes, the error matrix, along with UA, PA metrics, for the XGB classification using integrated S1 and S2 imagery, is shown in Table 5.

\begin{tabular}{|c|c|c|c|c|c|c|c|}
\hline \multirow{2}{*}{\multicolumn{2}{|c|}{$\begin{array}{c}\mathrm{S} 1+\mathrm{S} 2 \\
\text { single } \\
\text { date }\end{array}$}} & \multicolumn{5}{|c|}{ Reference data } & \multirow[b]{2}{*}{ UA } \\
\hline & & 1 & 2 & 3 & 4 & 5 & \\
\hline \multirow{5}{*}{. } & 1 & 11406 & 32 & 149 & 7 & 3 & 98.4 \\
\hline & 2 & 7 & 20846 & 1536 & 53 & 491 & 90.9 \\
\hline & 3 & 133 & 1597 & 19014 & 142 & 1347 & 85.5 \\
\hline & 4 & 34 & 1126 & 1744 & 7742 & 923 & 66.9 \\
\hline & 5 & 4 & 665 & 1873 & 30 & 11239 & 81.4 \\
\hline & PA & 98.5 & 85.9 & 78.2 & 97.1 & 80.3 & \\
\hline
\end{tabular}

Table 5. Error matrix for the XGB classification using integrated S1 and S2 imagery, with UA [\%] and PA [\%] for each class. 
As shown in Table 5, the water class achieved the highest UA and PA values of $98.4 \%$ and $98.5 \%$, respectively. Increased wetland classification accuracy has been reported in a paper from Kaplan and Avdan (2018). Also, for the S1 imagery, the VV polarization has a better effect on mapping calm water surfaces than VH polarization (Martinis et al., 2018). Slightly lower UA values were obtained for the bare soil and forest class. Overall, the forest class has the highest omission error of $21.8 \%$. According to Chust et al. (2004), the use of MT SAR imagery are more efficient for vegetation mapping, in opposite to single-date imagery. The built-up class attained higher PA values than UA values, which means that the XGB method correctly identified more ground truth pixels as bare soil, but the commission error was higher than the omission error. Including the Grey Level Co-occurrence Matrix (GLCM) texture variables increases classification results (Jin et al., 2018), especially for various built-up classes (Zakeri et al., 2017). Low vegetation class was mostly misclassified to the forest class, and viceversa. In order to increase LCC accuracy using a single-date S1 and S2 data, textural parameters (e.g., GLCM) for S1 (Idol et al., 2017), and various vegetation indices (e.g., NDVI, MSAVI) (Clerici et al., 2017) should be included and investigated along with machine learning methods used in this research.

\subsection{Land-cover classification on a multitemporal S1 and S2 imagery}

According to Vuolo et al. (2018), MT classification provides better results than single-date acquisitions within sub-optimal temporal windows. Therefore, available S2 imagery with zero cloud coverage before and after the reference date, as described in Table 2, were chosen for LCC. Afterward, according to S2 acquisition time, S1 imagery was used for MT LCC (Table 1). Accuracy assessment for the MT classification for S1 and S2 imagery, and using the combination of S1 and S2 are shown in Table 6 .

\begin{tabular}{|l|r|r|r|r|r|r|}
\hline Sensor & \multicolumn{2}{|c|}{ S1 } & \multicolumn{2}{c|}{ S2 } & \multicolumn{2}{c|}{ S1+S2 } \\
\hline Method & RF & XGB & RF & \multicolumn{1}{c|}{ XGB } & \multicolumn{1}{c|}{ RF } & XGB \\
\hline $\begin{array}{l}\text { OA } \\
{[\%]}\end{array}$ & 84.47 & 86.28 & 88.26 & 89.72 & 90.78 & 91.09 \\
\hline Kappa & 0.80 & 0.82 & 0.85 & 0.87 & 0.88 & 0.88 \\
\hline
\end{tabular}

Table 6. OA and Kappa values of RF and XGB, as applied to the multitemporal S1 and S2 imagery.

For the classification obtained using MT S1 imagery, XGB performed better than RF, with $\mathrm{OA}$ and Kappa values of $86.28 \%$ and 0.82 against $84.47 \%$ and 0.80 , respectively. MT S1 imagery significantly increased classification accuracy for RF and XGB method. Such improvement occurred due to spatial speckle filtering on the single-date S1 imagery, which was then stacked together. Maghsoudi et al. (2012) reported that MT filtering offers no advantage for classification tasks against spatial speckle filters. Therefore, the increase of OA metric of $14.06 \%$, and $14.26 \%$ for RF and XGB method on MT S1 imagery is achieved, respectively. In the MT S2 classification, XGB performed better than RF, with an OA value of $89.72 \%$, and $88.26 \%$, respectively. Obtained results for MT S2 imagery confirm that LCC methods applied to MT imagery perform better that single-date mapping methods (Belgiu and Csillik, 2018; Vuolo et al., 2018), since phenological patterns can be identified on a time-series datasets. Overall, the highest classification accuracy in this research was obtained using integrated MT S1 and S2 imagery. For the RF method, OA was $90.78 \%$, and Kappa 0.88, whereas the XGB method achieved
OA of $91.09 \%$, and Kappa 0.88. Sun et al. (2019) used S1, S2, and Landsat- 8 data for crop-type mapping. Their MT and the multi-source combination produced the highest OA of $93 \%$, and Kappa 0.91 with an RF classifier. According to the authors, although the use of S1 imagery affected the LCC, their ability to classify crop type was weaker than for S2 data. Viskovic et al. (2019) used MT S1 and S2 data for crop classification. RF outperformed other classifiers (e.g., SVM, K-nearest neighbors) with an OA of $84.20 \%$, and Kappa 0.82 . Furthermore, in order to compare and evaluate MT classification accuracy for separate land-cover classes, Table 7 shows the error matrix along with UA, PA metrics for the XGB classifier.

\begin{tabular}{|c|c|c|c|c|c|c|c|}
\hline \multirow{2}{*}{\multicolumn{2}{|c|}{$\begin{array}{c}\mathrm{S} 1+\mathrm{S} 2 \\
\text { multi } \\
\text { date }\end{array}$}} & \multicolumn{5}{|c|}{ Reference data } & \multirow[b]{2}{*}{ UA } \\
\hline & & 1 & 2 & 3 & 4 & 5 & \\
\hline \multirow{6}{*}{ 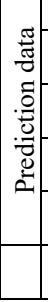 } & 1 & 11301 & 3 & 31 & 21 & 0 & 99.5 \\
\hline & 2 & 35 & 22466 & 1640 & 74 & 1073 & 88.8 \\
\hline & 3 & 218 & 631 & 20992 & 84 & 619 & 93.1 \\
\hline & 4 & 13 & 12 & 101 & 7762 & 31 & 98.0 \\
\hline & 5 & 23 & 1148 & 1517 & 46 & 12277 & 81.8 \\
\hline & PA & 97.5 & 92.6 & 86.5 & 97.2 & 87.7 & \\
\hline
\end{tabular}

Table 7. Error matrix for the XGB classification using integrated MT S1 and S2 imagery, with UA [\%], PA [\%] metrics for each class.

For the MT S1 and S2 classification, the water class achieved the highest UA and PA values of $99.5 \%$, and $97.5 \%$, respectively. Since no precipitation events occurred during the acquisition of the radar imagery, bare soil class was very well classified using MT data. Otherwise, soil moisture, which increases dielectric constant, has a major effect on the backscatter magnitude (Molijn et al., 2018). Regardless of LCC on a single-date or MT S1 and S2 imagery, the bare soil class achieved high PA results and was well classified on LCC maps. The highest increase from single-date to MT LCC, in terms of UA metric, achieved the built-up class. MT imagery significantly helped to correctly separate built-up areas, with an UA of $98.0 \%$, against UA of $66.9 \%$ obtained for LCC using single-date imagery. Using single speckle filtering on MT imagery significantly reduced confusion between forest and built-up class (i.e., 1744 false-negative forest pixels to 101 pixels). Besides MT imagery, GLCM texture features should be included in order to improve the classification OA, and the discrimination of urban areas (Dell'Acqua et al., 2003). In this research, the highest contribution of adding MT S1 and S2 imagery, in terms of decreased omission and commission errors, was for the forest and low vegetation class. For the forest class, UA and PA increased $7.6 \%$ and $8.3 \%$, respectively, whereas 1347 pixels misclassified as low vegetation decreased to 619 pixels. Using Frost spatial filter for speckle reduction for singledate S1 imagery, which were then stacked together, efficiently preserved edges and features, which alleviated the differentiation between forest and low vegetation class. Likewise, Rüetschi et al., (2018) showed that MT SAR imagery have the potential to supplement optical RS data for the mapping of mixed forests. Using them, monitoring at various spatial and temporal scales can be used for quantification of changes in species composition due to climate change. 


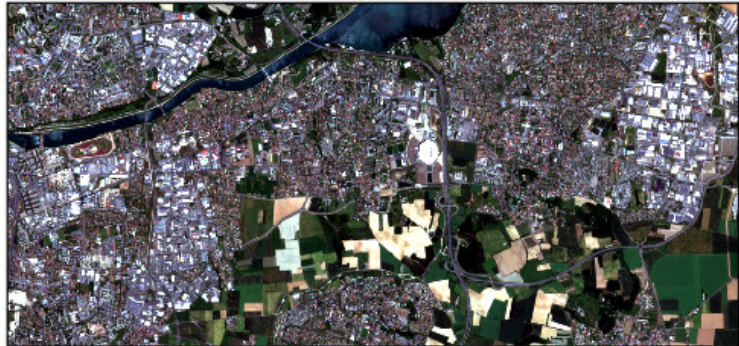

(a)

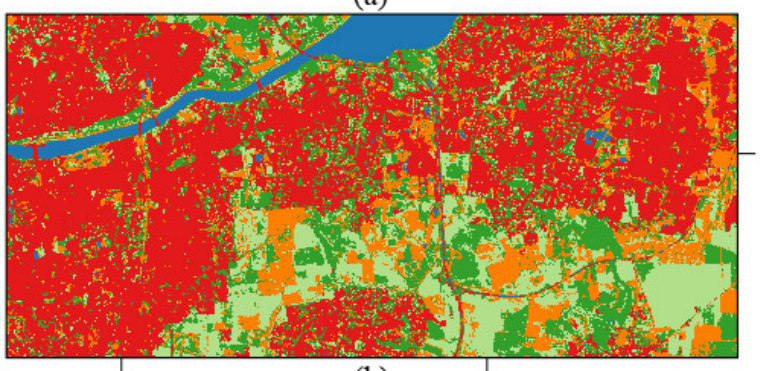

(b)

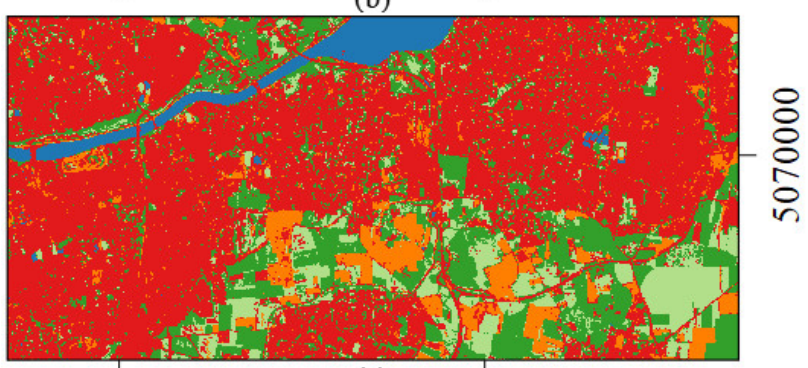

650000

(c)

655000

Projection: WGS 1984/UTM Zone 31 N; EPSG: 32631 Land-cover type

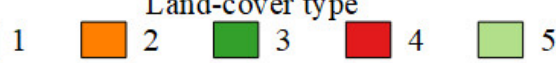

Figure 2. Example subset for a central part of the study area: (a) $\mathrm{S} 2$ 'true color' composite; (b) XGB classification using singledate S1 and S2 imagery; (c) XGB classification using MT S1 and S2 imagery.

Figure 2 shows classification maps obtained with S1 and S2 data using an XGB classifier. It can be seen that granular noise decreased with additional temporal features of S1 and S2 imagery. The major improvement for land-cover classification using MT imagery, occurred for the built-up class, because of stable objects like buildings, and other artificial built-up structures. Additionally, salt and pepper effect was reduced for forest and low vegetation class owing to the speckle filtering, and the MT classification map has sharper and clearer boundaries between the land-cover classes. Also, optical satellite imagery (i.e., S2) improved the distinction of different features (e.g., roads, still water), which are commonly misclassified using radar data because of similar backscatter pattern (Haas and Ban, 2017).

Chen and Guestrin (2016) reported that the XGB algorithm is designed for speed and performance by using gradient boosted decision trees, and hence, processing times for RF and XGB were investigated (Figure 3 ). The processing time for the singledate data using RF and XGB varied between $17 \mathrm{~min}$ and $31 \mathrm{~min}$ and between $3 \mathrm{~min}$ and $6 \mathrm{~min}$, respectively. Using MT imagery, the computational time varied between $20 \mathrm{~min}$ and $26 \mathrm{~min}$ for $\mathrm{RF}$, and between 5 min and 12 min for XGB.

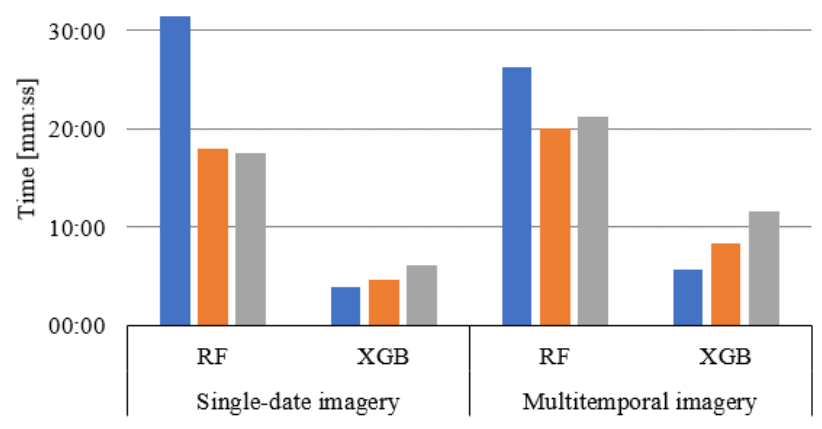

$\because \mathrm{S} 1=\mathrm{S} 2=\mathrm{S} 1+\mathrm{S} 2$

Figure 3. The computational time required for a single-date and MT classification using RF and XGB classifier.

This research evaluated the integration of multisource and multitemporal data provided by ESA for LCC. Regardless of LCC on a single-date or MT imagery, the highest classification results were achieved with integrated S1 and S2 imagery (Table 4 and Table 6). Gómez (2017) mentioned that the benefits of joining S1 and S2 data are more applicable for the pixel-based than in the polygon-based approach. Furthermore, classification accuracy significantly improved on MT SAR imagery, with an OA and Kappa increase of $14.06 \%$ and 0.19 , respectively. Temporal series of SAR imagery in combination with speckle filtering improves classification results, as reported in Skriver et al. (2011) and Maghsoudi et al. (2012). Future research should address the integration of GLCM texture features with MT SAR imagery, which can be used for areas that are most times covered with clouds. The second part of the research was to evaluate the RF and XGB classifiers for producing LCC maps. In this paper, for the pixel-based classification, XGB produced slightly better results than RF but outperformed it in terms of computational time. This gradient boosting algorithm gained popularity in various machine learning and data science competitions, and most recently in producing LCC maps (Man et al., 2018; Hirayama et al., 2019). Future research should compare the performance with different approaches, such as SVM, ANNs, and kernel-based extreme learning machine (KELM) (Clerici et al., 2017; Sonobe et al., 2017; Zhang et al., 2019).

\section{CONCLUSIONS}

In this research, classification accuracy was examined for LCC on multitemporal input data (S1, S2, and their integration) using two classifiers (RF and XGB).

A combination of multitemporal S1 and S2 imagery successfully classified five land-cover classes with the XGB classifier and OA of $91.09 \%$ and Kappa 0.88. Furthermore, the integration of MT imagery significantly improved the classification of urban areas and reduced misclassification between forest and low vegetation. It should also be noted how overall classification accuracy for S1 imagery increased from $72.02 \%$ to $86.28 \%$ with the use of the MT imagery, which can be useful for areas hampered by a frequent cloud cover. This research proved that RF and XGB algorithms are robust and can be used for LCC. In terms of computational time, XGB performed RF, whereas accuracy metrics were similar, so the trade-off between accuracy and processing time must be considered. 
This research evaluated the potential of radar and optical imagery for land-cover classification, so the future work should focus on specific applications (e.g., crop classification, vegetation monitoring, urban area mapping). Additionally, neural networks and deep learning methods should be examined for land-cover classification on remote sensing data.

\section{ACKNOWLEDGEMENTS}

This research is founded from the Croatian Science Foundation under Grant No. HRZZ-IP-2016-06-5621, the GEMINI project. Also, the University of Zagreb funded the RS4ENVIRO project entitled: "Advanced photogrammetry and remote sensing methods for environmental change monitoring" (Grant No. RS4ENVIRO) under which this research was conducted. The authors also thank the Ministry of Science and Education, Croatia, for supporting for space and space technology initiatives.

\section{REFERENCES}

Abdi, A.M., 2019. Land cover and land use classification performance of machine learning algorithms in a boreal landscape using Sentinel-2 data. GIScience \& Remote Sensing, pp. 1-20.

Abdullah, A.Y.M., Masrur, A., Gani Adnan, M.S., Al Baky, M.A., Hassan, Q.K., and Dewan, A., 2019. Spatio-temporal patterns of land use/land cover change in the heterogeneous coastal region of Bangladesh between 1990 and 2017. Remote Sensing, 11, pp. 1-28.

Belgiu, M., and Csillik, O., 2018. Sentinel-2 cropland mapping using pixel-based and object-based time-weighted dynamic time warping analysis. Remote Sensing of Environment, 204, pp. 509-523.

Breiman, L., 2001. Random forests. Machine Learning, 45(1), pp. 5-32.

Chen, T., and Guestrin, C., 2016. XGBoost: A Scalable Tree Boosting System. In Proceedings of the 22nd ACM Sigkdd International Conference on Knowledge Discovery and Data Mining, August 13-17, pp. 785-794.

Chust, G., Ducrot, D., Pretus, and J.L., 2004. Land cover discrimination potential of radar multitemporal series and optical multispectral images in a Mediterranean cultural landscape. International Journal of Remote Sensing, 25(17), pp. 3513-3528.

Clerici, N., Valbuena Calderón, C.A., and Posada, J.M., 2017. Fusion of sentinel-1a and sentinel-2A data for land cover mapping: A case study in the lower Magdalena region, Colombia. Journal of Maps, 13(2), pp. 718-726.

Dell'Acqua, F., Gamba, P., and Lisini, G., 2003. Improvements to urban area characterization using multitemporal and multiangle SAR images. IEEE Transactions on Geoscience and Remote Sensing, 41(9), pp. 1996-2004.

Dobrinić, D., Gašparović, M., and Župan, R., 2018. Horizontal Accuracy Assessment of PlanetScope, RapidEye and WorldView-2 Satellite Imagery. In: 18th International Multidisciplinary Scientific Geoconference SGEM 2018. 18, pp.
$129-136$.

Feng, Q., Yang, J., Zhu, D., Liu, J., Guo, H., Bayartungalag, B., and $\mathrm{Li}, \mathrm{B} ., 2019$. Integrating multitemporal Sentinel-1/2 data for coastal land cover classification using a multibranch convolutional neural network: A case of the Yellow River Delta. Remote Sensing, 11(9), pp. 1006.

Filipponi, F., 2019. Sentinel-1 GRD Preprocessing Workflow. In Multidisciplinary Digital Publishing Institute Proceedings, 18(1), pp. 11.

Foody, G., 2010. Assessing the Accuracy of Remotely Sensed Data: Principles and Practices, The Photogrammetric Record, 25(130), pp. 204-205.

Frost, V.S., Stiles, J.A., Shanmugan, K.S., and Holtzman, J.C., 1982. A Model for Radar Images and Its Application to Adaptive Digital Filtering of Multiplicative Noise. IEEE Transactions on pattern analysis and machine intelligence, (2), pp. $157-166$.

Gašparović, M., Dobrinić, D., and Medak, D., 2018. Urban Vegetation Detection Based on the Land-Cover Classification of Planetscope, Rapideye and Worldview-2 Satellite Imagery. In: 18th International Multidisciplinary Scientific Geoconference SGEM 2018. 18, pp. 249-256.

Gašparović, M., Dobrinić, D., and Medak, D., 2019. Geometric accuracy improvement of WorldView-2 imagery using freely available DEM data. The Photogrammetric Record, 34(167), pp. 266-281.

Gómez, M.G.C., 2017. Joint use of Sentinel-1 and Sentinel-2 for land cover classification: A machine learning approach. Lund University GEM thesis series.

Haas, J., and Ban, Y., 2017. Sentinel-1A SAR and sentinel-2A MSI data fusion for urban ecosystem service mapping. Remote Sensing Applications: Society and Environment, 8, pp. 41-53.

Hirayama, H., Sharma, R.C., Tomita, M., and Hara, K., 2019. Evaluating multiple classifier system for the reduction of saltand-pepper noise in the classification of very-high-resolution satellite images. International Journal of Remote Sensing, 40(7), pp. 2542-2557.

Idol, T., Haack, B., and Mahabir, R., 2017. Radar speckle reduction and derived texture measures for land cover/use classification: a case study. Geocarto International, 32(1), pp. 18-29.

Immitzer, M., Atzberger, C., and Koukal, T., 2012. Tree species classification with Random forest using very high spatial resolution 8-band worldView-2 satellite data. Remote Sensing, 4, pp. 2661-2693.

Jin, Y., Liu, X., Chen, Y., and Liang, X., 2018. Land-cover mapping using Random Forest classification and incorporating NDVI time-series and texture: a case study of central Shandong. International Journal of Remote Sensing, 39(23), pp. 87038723.

Kaplan, G., and Avdan, U., 2018. Sentinel-1 and Sentinel-2 data fusion for wetlands mapping: Balikdami, Turkey. International Archives of the Photogrammetry, Remote Sensing and Spatial Information Sciences - ISPRS Archives, 42, pp. 729-734. 
Kulkarni, V.Y., Petare, M., and Sinha, P.K., 2012. Analyzing Random Forest Classifier with Different Split Measures. In: Proceedings of the Second International Conference on Soft Computing for Problem Solving, December 28-30, 2012, pp. 691-699.

López-Caloca, A.A., Tapia-Silva, F.O., and Rivera, G., 2018. Sentinel-1 Satellite Data as a Tool for Monitoring Inundation Areas near Urban Areas in the Mexican Tropical Wet. In: Water Challenges of an Urbanizing World. IntechOpen.

Maghsoudi, Y., Collins, M.J., and Leckie, D., 2012. Speckle reduction for the forest mapping analysis of multi-temporal Radarsat-1 images. International Journal of Remote Sensing, 33(5), pp. 1349-1359.

Man, C.D., Nguyen, T.T., Bui, H.Q., Lasko, K., and Nguyen, T.N.T., 2018. Improvement of land-cover classification over frequently cloud-covered areas using landsat 8 time-series composites and an ensemble of supervised classifiers. International Journal of Remote Sensing, 39(4), pp. 1243-1255.

Martinis, S., Plank, S., and Ćwik, K., 2018. The use of Sentinel1 time-series data to improve flood monitoring in arid areas. Remote Sensing, 10(4), pp. 583.

Molijn, R., Iannini, L., López Dekker, P., Magalhães, P., and Hanssen, R., 2018. Vegetation Characterization through the Use of Precipitation-Affected SAR Signals. Remote Sensing, 10, pp. 1647.

Mountrakis, G., Im, J., and Ogole, C., 2011. Support vector machines in remote sensing: A review. ISPRS Journal of Photogrammetry and Remote Sensing, 66(3), pp. 247-259.

Niculescu, S., Talab Ou Ali, H., and Billey, A., 2018. Random forest classification using Sentinel-1 and Sentinel-2 series for vegetation monitoring in the Pays de Brest (France). In: Remote Sensing for Agriculture, Ecosystems, and Hydrology XX, 10783, pp. 1078305.

Noi, P.T., and Kappas, M., 2018. Comparison of random forest, k-nearest neighbor, and support vector machine classifiers for land cover classification using sentinel-2 imagery. Sensors, $18(1)$, pp. 18.

Oliver, C.J., 1991. Information from SAR images, Journal of Physics D: Applied Physics, 24(9), pp. 1493.

R Core Team. 2016. R: A Language and Environment for Statistical Computing. Vienna: R Foundation for Statistical Computing.

Rodriguez-Galiano, V.F., Ghimire, B., Rogan, J., Chica-Olmo, M., and Rigol-Sanchez, J.P., 2012. An assessment of the effectiveness of a random forest classifier for land-cover classification. ISPRS Journal of Photogrammetry and Remote Sensing, 67, pp. 93-104.

Rüetschi, M., Schaepman, M.E., and Small, D., 2018. Using multitemporal Sentinel-1 C-band backscatter to monitor phenology and classify deciduous and coniferous forests in Northern Switzerland. Remote Sensing, 10, pp. 1-30.

Shi, Z., and Fung, K.B., 1994. Comparison of digital speckle filters. In: International Geoscience and Remote Sensing
Symposium (IGARSS), pp. 2129-2133.

Skriver, H., Mattia, F., Satalino, G., Balenzano, A., Pauwels, V.R.N., Verhoest, N.E.C., and Davidson, M., 2011. Crop Classification Using Short-Revisit Multitemporal SAR Data. IEEE Journal of Selected Topics in Applied Earth Observations and Remote Sensing, 4(2), pp. 423-431.

Sonobe, R., Yamaya, Y., Tani, H., Wang, X., Kobayashi, N., Mochizuki, and Mochizuki, K. I., 2017. Assessing the suitability of data from Sentinel-1A and 2A for crop classification. GIScience \& Remote Sensing, 54(6), pp. 918938.

Souza, Jr, C., Siqueira, J., Sales, M., Fonseca, A., Ribeiro, J., Numata, I., Cochrane, M., Barber, C., Roberts, D., and Barlow, J., 2013. Ten-Year Landsat Classification of Deforestation and Forest Degradation in the Brazilian Amazon. Remote Sensing, 5(11), pp. 5493-5513.

Sun, C., Bian, Y., Zhou, T., and Pan, J., 2019. Using of multisource and multi-temporal remote sensing data improves croptype mapping in the subtropical agriculture region. Sensors, 19(10), pp. 1-23.

Torres, R., Snoeij, P., Geudtner, D., Bibby, D., Davidson, M., Attema, E., Potin, P., Rommen, B.Ö., Floury, N., Brown, M., Traver, I.N., Deghaye, P., Duesmann, B., Rosich, B., Miranda, N., Bruno, C., L'Abbate, M., Croci, R., Pietropaolo, A., Huchler, M., and Rostan, F., 2012. GMES Sentinel-1 mission. Remote Sensing of Environment, 120, pp. 9-24.

Van Tricht, K., Gobin, A., Gilliams, S., and Piccard, I., 2018. Synergistic Use of Radar Sentinel-1 and Optical Sentinel-2 Imagery for Crop Mapping: A Case Study for Belgium. Remote Sensing, 10(10), pp. 1642.

Veloso, A., Mermoz, S., Bouvet, A., Le Toan, T., Planells, M., Dejoux, J.F., and Ceschia, E., 2017. Understanding the temporal behavior of crops using Sentinel-1 and Sentinel-2-like data for agricultural applications. Remote Sensing of Environment, 199, pp. $415-426$.

Viskovic, L., Kosovic, I.N., and Mastelic, T., 2019. Crop classification using multi-spectral and multitemporal satellite imagery with machine learning. In: 2019 International Conference on Software, Telecommunications and Computer Networks (SoftCOM), pp. 1-5.

Vuolo, F., Neuwirth, M., Immitzer, M., Atzberger, C., and Ng, W.T., 2018. How much does multi-temporal Sentinel-2 data improve crop type classification? International Journal of Applied Earth Observation and Geoinformation, 72, pp. 122130.

Waske, B., and Braun, M., 2009. Classifier ensembles for land cover mapping using multitemporal SAR imagery. ISPRS Journal of Photogrammetry and Remote Sensing, 64(5), pp. 450-457.

Xiao, J., Li, J., and Moody, A., 2003. A detail-preserving and flexible adaptive filter for speckle suppression in SAR imagery. International Journal of Remote Sensing, 24(12), pp. 24512465 .

Zakeri, H., Yamazaki, F., and Liu, W., 2017. Texture Analysis and Land Cover Classification of Tehran Using Polarimetric 
Synthetic Aperture Radar Imagery. Applied Sciences, 7(5), pp. 452.

Zhang, C., Sargent, I., Pan, X., Li, H., Gardiner, A., Hare, J., and Atkinson, P.M., 2019. Joint Deep Learning for land cover and land use classification. Remote Sensing of Environment, 221, pp. 173-187.

Revised May 2020 